\title{
Um Relato Sobre a Experiência do Ensino de Programação para Crianças e Jovens de Forma Remota
}

\author{
Débora Zumpichiatti, Gabriel SantClair, João Victor Moreira, Janaina Gomide
}

\author{
${ }^{1}$ Engenharia - Universidade Federal do Rio de Janeiro, Campus Macaé \\ - Macaé - RJ - Brazil \\ \{deborazumpichiattil6, gsantclair, jvmoreira97, janainagomide\}@gmail.com
}

\begin{abstract}
The goals of this paper are: to propose a methodology for online minicourses of programming for children and tennagers, to present the courses that were created and to report the experience of the classes of these courses. The proposed methodology describes the interaction between the outreach project, the representatives of the partner school and its students. The main tools used were Youtube, Whatsapp, Google Forms and others specific to each course, all free. In total, 4 courses were proposed, 30 classes were held, reaching 509 students between 6 and 18 years old. The average views of the videoaulas and the answers obtained by the questionnaires reinforce the participation of students and also their satisfaction in each course.
\end{abstract}

\begin{abstract}
Resumo. Os objetivos desse artigo são: propor uma metodologia para o ensino online remoto de programação para crianças e jovens, apresentar os cursos que foram criados e relatar a experiência da realização das turmas desses cursos. A metodologia proposta descreve a interação entre o projeto de extensão, os representantes da escola parceira e seus alunos. As principais ferramentas utilizadas foram o Youtube, Whatsapp, Google Forms e outras especificas para cada curso, todas gratuitas. Ao total foram propostos 4 cursos, realizadas 30 turmas atingindo 509 alunos entre 6 e 18 anos. A média de visualizações das videoaulas e as respostas obtidas pelos questionários reforçam a participação dos alunos e também a satisfação dos mesmos em cada curso.
\end{abstract}

\section{Introdução}

O projeto de extensão Aprenda a Programar Jogando atua no desenvolvimento de atividades e divulgação do ensino da ciência da computação para crianças e jovens há 5 anos [Godinho et al. 2017]. No decorrer desse tempo diferentes cursos e oficinas foram estruturados e parcerias firmadas com escolas e outros projetos. Originalmente as atividades foram planejadas para serem realizadas de maneira presencial. Contudo, o ano de 2020 trouxe novos desafios, como a necessidade de se adaptar ao ensino remoto.

No método de ensino feito totalmente a distância e online, e-learning, a internet é apenas um dos componentes necessários. Nesse formato devem ser considerados os softwares que serão utilizados e quais atividades e práticas sociais envolvem o uso da internet nas escolas e em casa [Bruckman 2002]. As crianças e jovens de hoje em dia são acostumados a utilizar computador, tablet ou smartphone para interagirem com conteúdos e imagens na tela. Em [Sri and Krishna 2014] os autores citam os benefícios pedagógicos 
do ensino online. Há uma grande variedade de recursos educacionais online que fornecem um ambiente de diversão e interação no intuito de manter as crianças engajadas.

Diante da necessidade de adaptar as atividades do projeto ao ensino à distância foi necessário criar uma nova metodologia de ensino que viabilizasse o ensino de conceitos básicos de programação de forma remota envolvendo monitores, professores das instituições parceiras e seus alunos. Os objetivos desse artigo são: propor uma metodologia para o ensino online remoto de programação para crianças e jovens, apresentar os cursos de programação que foram criados e relatar a experiência da realização de 30 turmas desses cursos com participação de 509 alunos.

A metodologia proposta descreve a interação entre os monitores do projeto de extensão, os professores das escolas parceiras, que divulgam o curso, e seus alunos. $\mathrm{O}$ aplicativo WhatsApp é utilizado para comunicação e envio de materiais entre os monitores do projeto e a escola e o Youtube é utilizado para publicar as videoaulas produzidas pelos monitores do projeto. Essas ferramentas foram escolhidas por serem familiares a crianças, jovens e adultos e já terem sido utilizadas em experiências educacionais. Em [Martins and Gouveia 2019] os autores descrevem a experiência com o uso do WhatsApp para atividades extraclasse do ensino de programação e em [Santos et al. 2020] a utilização do Youtube para ensino da linguagem C em cursos de Engenharia.

Além da comunicação e divulgação dos materiais, as ferramentas de ensino de programação utilizadas são ferramentas online gratuitas que já são utilizadas nos cursos presenciais para crianças e jovens [Cafiero et al. 2018]. Algumas dessas ferramentas possibilitam criar jogos e aplicativos e são utilizadas também para ensinar professores a criarem seus aplicativos e ferramentas para usarem no ensino online. Em [da Silva Urzêda et al. 2020] foi relatado o ensino do Scratch ${ }^{1}$ para discentes de um curso de pedagogia de forma que eles aprendam a criar jogos e animações para utilizarem em sala de aula. Em [Pereira et al. 2020] o MIT App Inventor ${ }^{2}$ foi utilizado para ensinar professores do ensino fundamental a desenvolverem seus próprios aplicativos.

O desafio imposto pelas condições adversas do ano de 2020 se tornou uma oportunidade de levar as aulas para novos alunos, que não conheciam o projeto ou talvez não pudessem frequentar as aulas presenciais. Além do relato de experiência foi desenvolvido um catálogo de cursos ${ }^{3}$ contendo um compilado de informações de todos esses cursos desenvolvidos: onde encontrar as aulas, qual o público-alvo, materiais de apoio e outras informações. Esse material pode ser utilizado para divulgar nas escolas as possibilidades de cursos que podem ser desenvolvidos.

\section{Metodologia}

A metodologia desenvolvida envolve a comunicação entre o projeto, a escola parceira e os alunos. A Figura 1 mostra um fluxograma das tarefas realizadas durante o planejamento, execução e avaliação das atividades. Em seguida será detalhado como foi a divulgação do curso, a comunicação com as instituições parceiras, os materiais enviados, a interação entre os monitores das atividades e os alunos das escolas e a avaliação das atividades.

\footnotetext{
${ }^{1}$ Link ferramenta Scratch: https://scratch.mit.edu/

${ }^{2}$ Link MIT App Inventor: https://appinventor.mit.edu/

${ }^{3}$ Link catálogo de cursos: https://bit.ly/3cpAwbH
} 


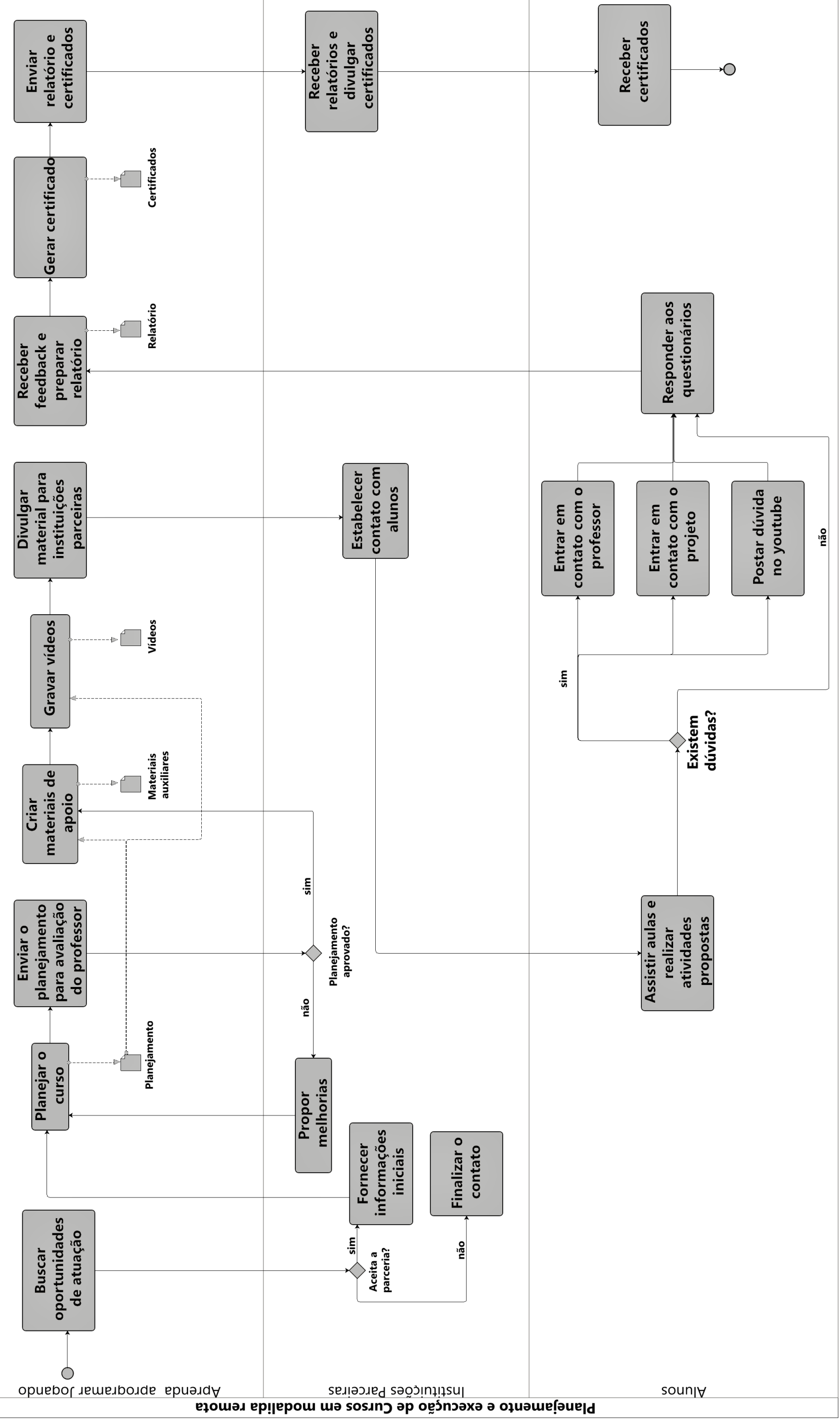

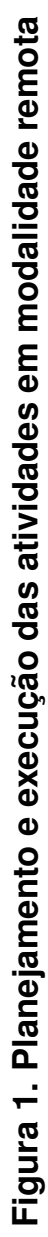




\subsection{Planejamento e Divulgação}

Para dar início a fase de planejamento, o primeiro passo foi entrar em contato com escolas e instituições parceiras, buscando entender a realidade atual dos colaboradores e as oportunidades de atuação. A equipe de monitores destinada a trabalhar com cada instituição tornou-se responsável pelo contato direto com professores, além de atuar no desenvolvimento, divulgação, realização e avaliação das atividades.

Através desse contato foi possível conhecer as necessidades e disponibilidades de equipamentos dos alunos. Assim, as atividades foram planejadas tendo em vista cada grupo específico. Para cada curso foram estabelecidos temas para as aulas, materiais auxiliares e exercícios que possibilitassem atender a demanda. Com a proposta de planejamento estruturada, iniciou-se a fase de validação, na qual todo o planejamento foi enviado para a instituição parceira para que esse passasse pela avaliação dos professores envolvidos. Sendo aprovado o planejamento, as estratégias de divulgação foram definidas e a fase de realização das atividades iniciada.

\subsection{Comunicação}

A comunicação com as escolas parceiras e os professores acontece durante todo o processo e foi mantida utilizando o aplicativo Whatsapp. Os monitores enviam todo o material, desde videoaulas até apostilas ou arquivos constando o código fonte para as aulas. A partir deste momento o material é levado aos alunos por meio dos professores através de diferentes plataformas definidas pela própria instituição, tendo em vista a acessibilidade de seu público. Durante o período de realização das atividades os monitores estavam sempre disponíveis para solucionar dúvidas a respeito do conteúdo, do material, das ferramentas aplicadas, entre outras.

Diferentemente das aulas presenciais, nas quais o contato entre monitor e aluno é constante em sala de aula, a modalidade remota impõe a barreira da distância. Para suprir tal falta de contato foram usadas as alternativas disponíveis nas diferentes plataformas utilizadas. Através do Youtube os alunos são incentivados a deixarem comentários com dúvidas, sugestões e respostas a perguntas propostas durante as aulas. As mídias sociais do projeto também foram utilizadas para comunicação entre monitor e aluno assim como e-mail para responder dúvidas e esclarecer informações diretas dos alunos ou professores.

\subsection{Materiais}

Os materiais enviados para os alunos são específicos para cada curso de acordo com o público-alvo. A seguir serão detalhados os tipos de materiais desenvolvidos:

- Playlist do Youtube: As aulas foram planejadas a fim de serem gravadas e postadas no canal oficial do projeto no Youtube ${ }^{4}$. Cada curso estabeleceu o tempo médio de duração das aulas e a abordagem estratégica para atrair seu público-alvo. Os cursos tiveram em média 7 vídeos com conteúdo teórico e prático.

- Apostila: Com o objetivo de fornecer apoio teórico aos alunos, foram desenvolvidas apostilas com conceitos básicos relacionados ao tema de cada curso.

- Arquivos do Google Colaboratory: O Google Colaboratory ${ }^{5}$ é uma plataforma que permite escrever e executar códigos em Python. Além de permitir que o usuário

\footnotetext{
${ }^{4}$ Link: https://youtube.com/c/AprendaProgramarJogando

${ }^{5}$ Link: https://colab.research.google.com/
} 
insira textos explicativos e execute diversas células de programação, a plataforma é online e gratuita. Nos cursos que utilizam a linguagem Python, o arquivo com o código desenvolvido é disponibilizado para os alunos.

- Link para Ferramentas: Algumas ferramentas online foram usadas como o Hour of Code $^{6}$, Compute it ${ }^{7}$, Scratch ${ }^{8}$ e MIT App Inventor ${ }^{9}$. Nesses casos, juntamente com os demais materiais, o link das ferramentas foi enviado para que o aluno acessasse e realizasse a atividade proposta.

- Materiais extras para Dinâmicas: algumas atividades desplugadas disponíveis em [Pinheiro et al. 2019] são realizadas nas videoaulas para introduzirem conceitos de programação. Os materiais necessários para a realização de tais atividades foram enviados aos alunos, como moldes para recortar e o passo a passo para realização das atividades.

\subsection{Avaliação}

A fim de acompanhar o aprendizado dos alunos e mapear novas necessidades do públicoalvo, a avaliação é feita de maneira contínua, por meio de formulários desenvolvidos no Google Forms especificamente para cada curso. Após finalizar o curso, a análise dos formulários gera relatórios com dados sobre a participação dos alunos, satisfação com o conteúdo e performance dos monitores. Esses relatórios são enviados aos professores das instituições parceiras juntamente com os certificados dos alunos.

\section{Planos de Curso}

Nessa seção serão apresentados cursos planejados, público-alvo, principais objetivos, linguagens de programação e ferramentas aplicadas, materiais auxiliares, plano de aulas e como realizar.

\subsection{Criação de Aplicativos}

O curso de Criação de Aplicativos foi desenvolvido para um público-alvo de jovens acima de 14 anos. Seu objetivo principal é ensinar conceitos básicos de programação computacional através do desenvolvimento de aplicativos de celular. A ferramenta usada é o MIT App Inventor não sendo necessário conhecimento prévio. O curso está disponível em uma playlist no Youtube ${ }^{10}$. Cada aula é compostas por 3 vídeos, nos quais são programadas as interfaces e blocos de aplicativo. A Tabela 1 mostra o plano de aulas sugerido.

\subsection{Programação para Crianças}

O curso de Programação para Crianças foi desenvolvido para um público-alvo de crianças entre 6 e 10 anos. Seu objetivo principal é introduzir conceitos básicos de programação computacional, através de atividades desplugadas, jogos e exemplos cotidianos. As ferramentas usadas são Hour of Code, Compute it, Scratch, Little Dot ${ }^{11}$, Silent Teacher ${ }^{12}$ e

\footnotetext{
${ }^{6}$ Link: https://hourofcode.com/br

${ }^{7}$ Link: https://compute-it.toxicode.fr/

${ }^{8}$ Link: https://scratch.mit.edu/

${ }^{9}$ Link: https://appinventor.mit.edu/

${ }^{10}$ Link: https://www.youtube.com/playlist?list=PLotgLi_FesFU0Jii1fM8FkK7jg6JnMnWd

${ }^{11}$ Link: https://little-dot.toxicode.fr/

${ }^{12}$ Link: https://silentteacher.toxicode.fr/
} 
Tabela 1. Plano de aulas do curso Criação de Aplicativos

\begin{tabular}{|c|c|c|l|}
\hline Semana & Tema & Materiais & Conteúdo \\
\hline $\mathbf{1}$ & Introdução & Vídeo & $\begin{array}{l}\text { Introdução ao curso e apresentação dos } \\
\text { monitores. }\end{array}$ \\
\hline $\mathbf{2}$ & Apresentação & $\begin{array}{c}\text { Vídeo e link MIT } \\
\text { App Inventor }\end{array}$ & $\begin{array}{l}\text { Apresentação do MIT App Inventor e } \\
\text { suas principais funcionalidades. }\end{array}$ \\
\hline $\mathbf{3}$ & $\begin{array}{c}\text { App: Conta de } \\
\text { Banco }\end{array}$ & $\begin{array}{c}\text { 3 vídeos e link } \\
\text { MIT App Inventor }\end{array}$ & $\begin{array}{l}\text { Apresentação das ferramentas de inter- } \\
\text { face e programação. Desenvolvimento da } \\
\text { interface e blocos do primeiro aplicativo. }\end{array}$ \\
\hline $\mathbf{4}$ & $\begin{array}{c}\text { App: Pegue a } \\
\text { Toupeira }\end{array}$ & $\begin{array}{c}\text { 3 vídeos e link } \\
\text { MIT APP Inventor }\end{array}$ & $\begin{array}{l}\text { Desenvolvimento da interface e blocos do } \\
\text { segundo aplicativo }\end{array}$ \\
\hline
\end{tabular}

Kahoot $^{13}$ não sendo necessário conhecimento prévio de programação. O curso está disponível em uma playlist no Youtube ${ }^{14}$ e cada aula é dividida em duas partes, sendo uma teórica e a outra prática. A Tabela 2 apresenta o plano de aulas sugerido.

Tabela 2. Plano de aulas do curso Programação para Crianças

\begin{tabular}{|c|c|c|l|}
\hline Semana & Tema & Materiais & Conteúdo \\
\hline $\mathbf{1}$ & Introdução & Vídeo & $\begin{array}{l}\text { Introdução ao curso e apresentação dos } \\
\text { monitores. }\end{array}$ \\
\hline $\mathbf{2}$ & Algoritmo & $\begin{array}{c}\text { Vídeo e link Hour } \\
\text { of Code }\end{array}$ & $\begin{array}{l}\text { Explicação e exemplo cotidiano do con- } \\
\text { ceito e aplicação usando a ferramenta. }\end{array}$ \\
\hline $\mathbf{3}$ & $\begin{array}{c}\text { Movimentos e } \\
\text { Repetição }\end{array}$ & $\begin{array}{c}\text { Vídeo e link } \\
\text { Compute it }\end{array}$ & $\begin{array}{l}\text { Explicação teórica, exemplo do conceito } \\
\text { e aplicação usando a ferramenta. }\end{array}$ \\
\hline $\mathbf{4}$ & $\begin{array}{c}\text { Condicionais e } \\
\text { Booleanos }\end{array}$ & $\begin{array}{c}\text { Vídeo e link Little } \\
\text { Dot }\end{array}$ & $\begin{array}{l}\text { Explicação teórica, exemplo através de } \\
\text { dinâmica desplugada e aplicação usando } \\
\text { a ferramenta. }\end{array}$ \\
\hline $\mathbf{5}$ & Variáveis & $\begin{array}{c}\text { Vídeo e link Silent } \\
\text { Teacher }\end{array}$ & $\begin{array}{l}\text { Explicação teórica, exemplos do conceito } \\
\text { e aplicação usando a ferramentar. }\end{array}$ \\
\hline $\mathbf{6}$ & Revisão & Vídeo & Revisão dos conceitos e Quiz no Kahoot \\
\hline $\mathbf{7}$ & Encerramento & Vídeo & $\begin{array}{l}\text { Criação de jogo do Scratch usando os } \\
\text { conceitos abordados ao longo do curso. }\end{array}$ \\
\hline
\end{tabular}

\subsection{Python + Matemática}

O curso Python + Matemática foi desenvolvido para um público-alvo de jovens acima de 14 anos. Seu objetivo principal é unir a matemática a conceitos básicos de programação computacional, mostrando a seus participantes que os conceitos da matemática podem se tornar mais simples usando programação. A linguagem de programação usada é o Python, não sendo necessário qualquer conhecimento prévio. O curso está disponível em uma playlist no Youtube ${ }^{15}$ e cada aula é composta por um vídeo teórico e um vídeo com implementação de código e solução de exercícios. Essa atividade pode ser realizada em formato de curso ou oficina. Para realizar o curso basta seguir o plano de aulas sugerido na

\footnotetext{
${ }^{13}$ Link: https://kahoot.com/

${ }^{14}$ Link: https://www.youtube.com/playlist?list=PLotgLi_FesFWZvoljLReMkFipI6prnasM

${ }^{15}$ Link: https://www.youtube.com/playlist?list=PLotgLi_FesFVQcnXEH2dpkgmxrgGUukfC
} 
Tabela 3. Para realizar a oficina basta selecionar o tema desejado e executar as atividades propostas para o mesmo.

Tabela 3. Plano de aulas do curso Python + Matemática

\begin{tabular}{|c|c|c|l|}
\hline Semana & Tema & Materiais & Conteúdo \\
\hline $\mathbf{1}$ & Introdução & Vídeo & $\begin{array}{l}\text { Introdução ao curso, apresentação dos mo- } \\
\text { nitores e introdução ao Google Colabora- } \\
\text { tory e suas funcionalidades. }\end{array}$ \\
\hline $\mathbf{2}$ & $\begin{array}{c}\text { Progressão } \\
\text { Aritmética }\end{array}$ & $\begin{array}{c}2 \text { vídeos, arquivo } \\
\text { Google Colaboratory e } \\
\text { apostila }\end{array}$ & $\begin{array}{l}\text { Introdução ao tema e explicação teórica so- } \\
\text { bre comandos de entrada e saída, variáveis } \\
\text { e repetição (while) no Python. Resolução } \\
\text { de exercícios. }\end{array}$ \\
\hline $\mathbf{3}$ & Estatística & $\begin{array}{c}2 \text { vídeos, arquivo } \\
\text { Google Colaboratory e } \\
\text { apostila }\end{array}$ & $\begin{array}{l}\text { Introdução ao tema e explicação teórica so- } \\
\text { bre repetição (for) no Python. Resolução } \\
\text { de exercícios. }\end{array}$ \\
\hline $\mathbf{4}$ & Matemática \\
Financeira & $\begin{array}{c}\text { Gódeos, arquivo } \\
\text { Google Colaboratory e } \\
\text { apostila }\end{array}$ & $\begin{array}{l}\text { Introdução ao tema e explicação teórica } \\
\text { sobre operações matemáticas e comandos } \\
\text { condicionais no Python. Resolução de } \\
\text { exercícios. }\end{array}$ \\
\hline
\end{tabular}

\subsection{Python para Garotas Cientistas}

O curso Python para Garotas Cientistas foi desenvolvido para um público-alvo de crianças e jovens. Criado em parceria com o projeto de extensão <omitido para revisão $>$, seu objetivo principal é propagar o ensino de programação de computadores de maneira simples, integrando conceito básicos da matemática à programação. Os temas abordados nessa atividade foram propostos por professores de escolas públicas com o objetivo de desenvolver diferentes cursos que alcancem faixa etárias específicas. Foram planejados quatro cursos cujos temas são "Operações básicas e divisão com resto", "Divisão com inteiros e racionais", "Função afim" e "Sequência numérica", direcionados ao $6^{\circ}$ e $7^{\circ}$ ano do ensino fundamental e ao $1^{\circ}$ e $2^{\circ}$ ano do ensino médio, respectivamente. A linguagem de programação utilizada é o Python, não sendo necessário qualquer conhecimento prévio. As aulas estão disponíveis em uma playlist no Youtube ${ }^{16}$ e, para realizar o curso, basta selecionar o tema que melhor se aplica ao público desejado e executar as atividades do plano de aulas - atividades essas válidas para qualquer dos temas. As aulas 1 e 4 são comuns a todos os cursos e na Tabela 4 será detalhado o plano de aulas.

\section{Resultados}

Os resultados alcançados estão resumidos na Tabela 5. Foram realizadas 30 turmas dos quatro cursos criados e um total de 509 alunos participaram desses cursos. A idade dos alunos variou de 6 a 18 anos e o curso com maior média de visualizações foi o curso de Criação de Aplicativos.

O curso de Criação de Aplicativos foi realizado em parceria com uma instituição social para qualificação profissional. Foram ofertadas 12 turmas e 359 alunos participaram das atividades, sendo 148 homens e 211 mulheres. No total, os vídeos desse curso

\footnotetext{
${ }^{16}$ Link: https://www.youtube.com/playlist?list=PLotgLi_FesFVbo8_mWsbIhQ3jucC_SN20
} 
Tabela 4. Plano de aulas do curso Python para Garotas Cientistas

\begin{tabular}{|c|c|c|l|}
\hline Semana & Tema & Materiais & Conteúdo \\
\hline $\mathbf{1}$ & Introdução & Vídeo e apostila & $\begin{array}{l}\text { Introdução ao curso e apresentação dos } \\
\text { monitores. }\end{array}$ \\
\hline $\mathbf{2}$ & Aula teórica & Vídeo e apostila & $\begin{array}{l}\text { Introdução teórica sobre o tema proposto } \\
\text { e aplicação no Python. }\end{array}$ \\
\hline $\mathbf{3}$ & $\begin{array}{c}\text { Resolução de } \\
\text { exercícios }\end{array}$ & Vídeo & Resolução de exercícios sobre o tema. \\
\hline $\mathbf{4}$ & Revisão & Vídeo & $\begin{array}{l}\text { Revisão dos conceitos abordados e encer- } \\
\text { ramento. }\end{array}$ \\
\hline
\end{tabular}

Tabela 5. Cursos remotos realizados.

\begin{tabular}{|l|c|c|c|c|}
\hline Curso & $\begin{array}{c}\text { Quantidade } \\
\text { de Turmas }\end{array}$ & $\begin{array}{c}\text { Quantidade } \\
\text { de Alunos }\end{array}$ & $\begin{array}{c}\text { Média de } \\
\text { Visualizações }\end{array}$ & Idade \\
\hline Criação de Aplicativos & 12 & 359 & 583 & $14-18$ anos \\
\hline Programação para Crianças & 5 & 51 & 98 & $6-10$ anos \\
\hline Python + Matemática & 8 & 55 & 68 & $14-18$ anos \\
\hline $\begin{array}{l}\text { Python para Garotas Cien- } \\
\text { tistas }\end{array}$ & 5 & 44 & 72 & $14-18$ anos \\
\hline Total & $\mathbf{3 0}$ & $\mathbf{5 0 9}$ & - & - \\
\hline
\end{tabular}

tiveram mais de 4600 visualizações, sendo o máximo de visualizações em um único vídeo igual a 708 .

O curso de Programação para Crianças foi desenvolvido em parceria com uma escola pública, sendo ofertado para 5 turmas do terceiro ao quinto ano do Ensino Fundamental. Ao todo, participaram das atividades 51 alunos, sendo 25 homens e 26 mulheres. No total, os vídeos desse curso tiveram 588 visualizações, sendo o máximo de visualizações em um único vídeo igual a 175. É importante ressaltar que o encontro da Semana 7, descrito na Tabela 2 do presente artigo, não foi realizado para as turmas descritas acima devido às mudanças ocorridas no calendário durante a aplicação do curso.

No curso de Python + Matemática foram ofertadas 8 turmas em parceria com uma escola pública. Ao todo, participaram das atividades 55 alunos, sendo 33 homens e 22 mulheres. No total, os vídeos desse curso tiveram mais de 480 visualizações, sendo o máximo de visualizações em um único vídeo igual a 151.

No curso de Python para Garotas Cientistas foram ofertadas 5 turmas, todas em colaboração com um projeto parceiro e com 3 escolas públicas. Ao todo, participaram das atividades 44 alunos, sendo 17 homens e 27 mulheres. No total, os vídeos tiveram mais de 790 visualizações, sendo que o vídeo com maior visualização teve 223 acessos.

\section{Desafios Encontrados e Lições Aprendidas}

O primeiro desafio encontrado foi em relação à produção de conteúdo audiovisual e a qualidade desse conteúdo. A grande maioria dos monitores do projeto não possuía experiência com a produção de videoaulas, portanto foi necessário um período de adaptação 
para que os mesmos pudessem entender quais equipamentos utilizar para a captação de áudio e vídeo, quais programas para a captação da tela e do material das aulas e como realizar uma boa edição para manter a coesão do conteúdo a ser passado para os alunos.

Um segundo desafio, encontrado no curso de Programação para Crianças, foi em relação à acessibilidade dos alunos a alguns dos jogos propostos. Os jogos Compute it e Little dot são acessíveis somente pelo computador. Entretanto, alguns dos alunos estavam acompanhando as aulas por smartphones e não tinham acesso ao computador, o que acabou impedindo o acesso a essas ferramentas. Como solução os monitores sugeriram outros jogos com temáticas semelhantes que podem ser acessados também pelo celular.

Por fim, um terceiro e último desafio encontrado é relacionado ao engajamento de algumas das turmas com o curso proposto. Alguns alunos acabaram perdendo o interesse, devido à pouca interação direta com os monitores do projeto, visto que a grande maioria dos cursos era disponibilizada somente de forma assíncrona através dos vídeos disponibilizados no Youtube.

Em relação às lições aprendidas podemos destacar a interação entre monitores e alunos. Foi observado que nas turmas onde foram realizadas reuniões online via Google Meet os alunos permaneceram engajados durante o andamento do curso. Dessa forma concluiu-se que, no futuro, essas reuniões podem ser planejadas com uma maior frequência, ou até mesmo uma interação em lives feitas através do YouTube, como forma de motivar os alunos e manter um contato mais direto.

Outra observação foi feita quanto ao formato da avaliação dos alunos no curso de Programação para Crianças. Notou-se que os formulários desenvolvidos no Google Forms não foram eficazes para esse público alvo. Apesar das crianças assistirem as videoaulas e comentarem no Youtube a maioria não preencheu o formulário. Uma possível solução seria interagir mais por meio dos comentários dos vídeos no YouTube ou até mesmo em encontros síncronos com as turmas.

\section{Conclusão}

Este artigo apresentou um relato de experiência sobre o planejamento, execução, resultados e lições aprendidas dos cursos remotos: Criação de Aplicativos, Programação para Crianças, Python + Matemática e Python para Garotas Cientistas. Sendo os objetivos: relatar a metodologia aplicada nesses cursos, como alternativa para o ensino online à distância de programação para crianças e jovens, e apresentar como cada um deles foi desenvolvido.

A metodologia foi implementada com sucesso nos 4 cursos apresentados e alcançou um total de 509 alunos divididos em 30 turmas, dentre eles estão inclusos crianças e jovens na faixa etária de 6 aos 18 anos. A média de visualizações em cada curso reforça o sucesso de cada um, visto que essa representa uma quantidade maior de visualizações por vídeo do que a quantidade de alunos naquele curso.

Como pontos positivos desse método de ensino online temos o alcance que o mesmo pode proporcionar, atingindo jovens e crianças que, numa situação normal de aulas presenciais, não conseguiriam estar presentes. Além disso, a disponibilidade dos cursos através de uma plataforma tão acessível como é o YouTube aumenta a visibilidade do projeto, tornando-o uma das referências de ensino da programação computacional para 
crianças e jovens na região em que se encontra e, futuramente, em nível nacional.

Como pontos negativos dessa metodologia destaca-se a pouca interação entre alunos e monitores e a limitação de alguns alunos quanto ao acesso a computadores. Poucos alunos reportaram dúvidas por meio de comentários nas videoaulas e o contato com os monitores aconteceu em aulas síncronas, que foram esporádicas. A limitação ao acesso a computadores implicou em uma adaptação das ferramentas para que essas possam ser acessadas em qualquer aparelho (dentre tablets, celulares e computadores).

Por fim, além de aprimorar os cursos já desenvolvidos, tem-se como perspectivas para o futuro a criação de novos cursos, expandindo o alcance dessas aulas através de novas temáticas e assuntos relacionados à programação computacional. Também é esperado um desenvolvimento maior na interação entre alunos e monitores, através de lives e reuniões, para a obtenção de um feedback mais direto e de uma maior proximidade entre as duas partes, como forma de motivar ainda mais os alunos que participarem dos cursos.

\section{Referências}

Bruckman, A. (2002). The future of e-learning communities. Commun. ACM, 45(4):60-63.

Cafiero, C., Pinheiro, J. V., and Gomide, J. (2018). Avaliação das ferramentas utilizadas em um curso de programação para crianças: Percepções dos alunos e seus instrutores. In Anais do Workshop de Informática na Escola, volume 24, page 51.

da Silva Urzêda, R. F., Severiano, E. P. R., and dos Santos Amorim, L. (2020). O uso do scratch no curso de pedagogia: relato de uma experiência interdisciplinar. In Anais do XXVIII Workshop sobre Educação em Computação, pages 21-25. SBC.

Godinho, J., Torres, K., Batista, G., Andrade, E., and Gomide, J. (2017). Projeto aprenda a programar jogando: Divulgando a programação de computadores para crianças e jovens. In Anais do XXV Workshop sobre Educação em Computação. SBC.

Martins, E. and Gouveia, L. (2019). Uso do whatsapp em atividades educativas extraclasse on-line no ensino de programação. In Anais do XXVII Workshop sobre Educação em Computação, pages 141-150. SBC.

Pereira, F. G. H. S., de Araújo, G. S., Cheung, L. M., de Araújo, A. V., and Zunta, H. B. (2020). Relato da utilização da plataforma app inventor como ferramenta de ensino de lógica de programação para professores da rede básica de ensino. In Anais do XXVIII Workshop sobre Educação em Computação, pages 86-90. SBC.

Pinheiro, J., Godinho, J., Guedes, Y., Cardoso, G., Zumpichiatti, D., and Gomide, J. (2019). Programa (ação): Atividades lúdicas para ensino de programação em escolas públicas. In Anais do XXVII Workshop sobre Educação em Computação, pages 91100. SBC.

Santos, L. d. C., da Silva, S. S., and de Meireles, S. P. (2020). Utilizando o youtube para ensino da linguagem c em cursos de engenharia. In Anais do XXVIII Workshop sobre Educação em Computação, pages 156-160. SBC.

Sri, K. U. and Krishna, T. V. (2014). E-learning: Technological development in teaching for school kids. International Journal of Computer Science and Information Technologies, 5(5):6124-6126. 